\title{
Crack velocity dependent toughness in rate dependent materials
}

\author{
Chad M. Landis ${ }^{\mathrm{a}, *}$, Thomas Pardoen ${ }^{\mathrm{a}, \mathrm{b}}$, John W. Hutchinson ${ }^{\text {a }}$ \\ a Division of Engineering and Applied Science, Harvard University, Cambridge, MA 02138, USA \\ b Département des Sciences des Matériaux et Procédés, Université catholique de Louvain, PCIM, \\ 2 Place Sainte Barbe, 1348 Louvain-la-Neuve, Belgium
}

Received 22 May 2000

\begin{abstract}
Mode I, quasi-static, steady-state crack growth is analyzed for rate dependent materials under plane strain conditions in small scale yielding. The solid is characterized by an elastic-viscoplastic constitutive law and the plane ahead of the crack tip is embedded with a rate dependent fracture process zone. The macroscopic work of fracture of the material is computed as a function of the crack velocity and the parameters characterizing the fracture process zone and the solid. With increasing crack velocity a competition exists between the strain rate hardening of the solid, which causes elevated tractions ahead of the crack tip that tend to drive crack propagation, and the rate strengthening of the fracture process zone which tends to resist fracture. Results for material parameters characteristic of polymers show that the toughness of the material can either increase or decrease with increasing crack velocity. To motivate the model, the cohesive zone parameters are discussed in terms of failure mechanisms such as crazing and void growth ahead of the crack tip. The toughness of rubber modified epoxies is explained by employing the fracture model along with micromechanical void cell calculations. (c) 2000 Elsevier Science Ltd. All rights reserved.
\end{abstract}

Keywords: Steady crack growth; Fracture toughness; Viscoplasticity; Cohesive zones; Polymers; Void growth

\section{Introduction}

This paper focuses on the mechanics of steadystate crack propagation in strain rate dependent materials. Materials which can be described by a simple elastic-visoplastic constitutive law will be investigated. Emphasis is placed on slow crack

\footnotetext{
${ }^{*}$ Corresponding author. Present address: Department of Mechanical Engineering and Material Science, Rice University, MS 321, P.O. Box 1892, Houston, TX 77251, USA. Tel.: +1713-348-3609; fax: +1-713-348-5423.

E-mail address: landis@rice.edu (C.M. Landis).
}

growth $\left(\dot{a} / c_{\mathrm{s}} \approx 10^{-6}\right)$ such that inertial effects are negligible. Even in this regime of crack velocities, the rate dependence of several polymer systems is of sufficient sensitivity to cause the material toughness to depend on crack speed. Recent experiments by Du et al. (2000) on rubber-modified epoxy and Sener (1999) on epoxy adhesives have shown that the fracture toughness can double or half with a 5-10 fold increase in crack velocity. To further complicate matters, the fracture toughness does not have a unique trend with respect to the crack velocity. As discussed in detail by Webb and Aifantis (1995), there can exist certain regimes of behavior where toughness increases with crack 
velocity and others where toughness decreases in a given material. Our primary goal is to derive general predictions about the material parameters that dictate these trends. The second is to motivate the analysis by referring to two well-defined systems, i.e. failure by crazing in amorphous polymers and by cavitation in rubber modified epoxies or polyamides.

Fig. 1 is a schematic of a crack propagating in an elastic-plastic solid under Mode I, plane strain, small scale yielding, steady-state conditions. The displayed shape of the plastic zone is the result of a calculation for an elastic-perfectly plastic, rate independent material. Far from the crack tip the stresses follow the Mode I elastic $K$ field. As the crack propagates through the solid, the crack tip is surrounded by active plasticity, elastic unloading and plastic reloading sectors (Drugan et al., 1982). Far behind the crack tip there is a wake of residual plastic strains where the material has unloaded elastically.
For any steady-state fracture process the macroscopic energy release rate, $\Gamma_{\mathrm{ss}}$, can be written as $\Gamma_{\mathrm{ss}}=\Gamma_{\mathrm{f}}+\Gamma_{\mathrm{p}}$,

where $\Gamma_{\mathrm{f}}$ represents the work of separation for the near tip fracture process and $\Gamma_{\mathrm{p}}$ represents the extrinsic toughening contribution from plastic dissipation and stored elastic energy in the wake of residual plastic strains. At the simplest level the fracture process can be modeled by a one-dimensional (i.e. no multiaxial loading effects) tractionseparation law which is embedded on the plane ahead of the crack tip as depicted in Fig. 1. The extrinsic contribution to the toughness depends on the constitutive behavior of the bulk solid. Given the bulk and fracture process properties, $\Gamma_{\mathrm{ss}}$ can be computed numerically via the finite element method. Embedded process zone (EPZ) models of this type have been used to quantify the relationship between $\Gamma_{\mathrm{ss}}$ and material parameters for conventional elastic-plastic solids, strain gradient

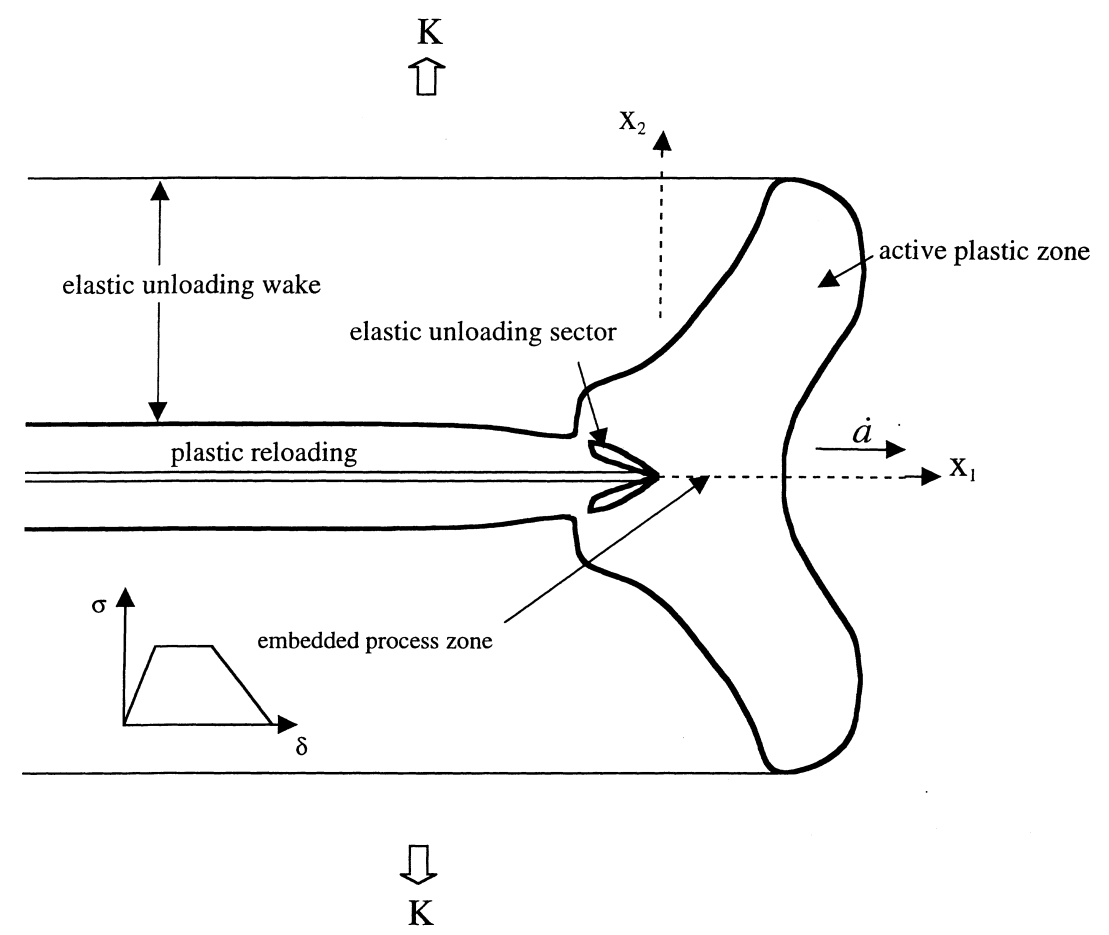

Fig. 1. Computed shape of the plastic zone around a crack growing steadily in an elastic-perfectly plastic solid under small scale yielding, Mode I loading conditions. 
elastic-plastic solids and ductile thin film/substrate systems (Tvergaard and Hutchinson, 1992; Wei and Hutchinson, 1997a,b).

Tvergaard and Hutchinson (1992) have shown that the significant parameters controlling the traction-separation law are the peak stress, $\hat{\sigma}$, and $\Gamma_{\mathrm{f}}$. The specific shape of the traction-separation law is of secondary importance. Their work has demonstrated that there is a significant "leveraging" effect on $\Gamma_{\mathrm{ss}}$ if $\hat{\sigma}$ is of sufficient magnitude. For example, in an elastic-perfectly plastic solid, if $\hat{\sigma}<2.5 \sigma_{y}$, then significant plastic dissipation is not able to occur and $\Gamma_{\mathrm{ss}}$ is only slightly greater than $\Gamma_{\mathrm{f}}$. However, if $\hat{\sigma}>3 \sigma_{y}$, then $\Gamma_{\text {ss }}$ is unbounded since the bulk solid cannot produce tractions greater than $3 \sigma_{y}$ on the plane ahead of the crack tip. In strain hardening plastic solids $\Gamma_{\mathrm{ss}}$ is always finite but dramatic increases in toughness occur in the range of $\hat{\sigma}=3-5 \sigma_{y}$. Generally, hardening of the solid by accumulated plastic strain, strain gradient effects or strain rate effects postpones the elevation of $\Gamma_{\text {ss }}$ to higher values of $\hat{\sigma}$.

The class of materials we will consider in this paper harden as the effective plastic strain rate increases. This implies that a fast growing crack will have higher tractions acting on the plane of separation than a slower crack. Therefore, faster crack velocities will have a tendency to drive the fracture process more readily. At first glance this suggests that $\Gamma_{\mathrm{ss}}$ decreases with increasing crack speed, and we will show that this is the case when the fracture process is rate independent. However, it is likely that a rate dependent material will also have a rate dependent fracture process. A classical example is the crazing process in glassy polymers (Kramer and Berger, 1990), and we could also envision a rate dependent void growth and coalescence process in rubber modified epoxies. The addition of rate dependence to the fracture process further complicates the model, not only do we need to determine $\hat{\sigma}$ and $\Gamma_{\mathrm{f}}$ but we also need to identify how these quantities vary with crack opening rate. We will propose a simple form for the rate dependence of the traction-separation law. The two new parameters that we introduce in the fracture process zone, the rate exponent $q$ and the characteristic opening rate $\dot{\delta}_{\mathrm{o}}$, will be shown to significantly effect the $\Gamma_{\mathrm{ss}}$ vs $\dot{a}$ behavior of the material. Changes in these parameters can be associated with the propensity for "rate strengthening" of the fracture process zone. The rate strengthening of the cohesive zone law introduces a competition between the rate hardening of the bulk solid, which tends to enhance crack growth and the rate strengthening of the fracture process zone, which resists propagation.

\section{Rate dependent constitutive law of the solid}

We adopt a rate dependent elastic-viscoplastic constitutive model of the form used by Marusich and Ortiz (1995) and Xia and Shih (1995):

$$
\begin{aligned}
& \left(1+\frac{\dot{\bar{\varepsilon}} \mathrm{p}}{\dot{\varepsilon}_{0}}\right)=\left(\frac{\bar{\sigma}}{\sigma_{y}}\right)^{m} \text { if } \bar{\sigma} \geqslant \sigma_{y}, \\
& \dot{\bar{\varepsilon}}^{\mathrm{p}}=0 \quad \text { if } \bar{\sigma}<\sigma_{y},
\end{aligned}
$$

where $\bar{\sigma}=\sqrt{(3 / 2) s_{i j} s_{i j}}$ is the effective stress, $s_{i j}=\sigma_{i j}-(1 / 3) \sigma_{k k} \delta_{i j}$ is the deviatoric stress tensor, $\sigma_{y}$ the static tensile yield strength,

$\dot{\bar{\varepsilon}} \mathrm{p}=\sqrt{(2 / 3) \dot{\varepsilon}_{i j}^{\mathrm{p}} \dot{\varepsilon}_{i j}^{\mathrm{p}}}$

is the effective plastic strain rate, $\dot{\varepsilon}_{0}$ a reference plastic strain rate and $m$ is the plastic strain rate sensitivity exponent. In general the static tensile yield strength may depend on the accumulated plastic strain. However, being primarily concerned with rate effects, we will assume perfect plasticity for static loading and hence $\sigma_{y}$ is a constant. This form of the constitutive law was chosen over the form $\bar{\sigma} / \sigma_{y}=1+\left(\dot{\bar{\varepsilon}}^{\mathrm{p}} / \dot{\varepsilon}_{0}\right)^{n}$ due to a more favorable fit with uniaxial tension data obtained from Sener (1999) on an epoxy resin. The components of the plastic strain rate are then given by

$\dot{\varepsilon}_{i j}^{\mathrm{p}}=\frac{3}{2} \dot{\bar{\varepsilon}} \mathrm{p} \frac{s_{i j}}{\bar{\sigma}}$.

Finally, the elastic strain rates are given by

$\dot{\varepsilon}_{i j}^{\mathrm{e}}=\frac{1+v}{E} \dot{\sigma}_{i j}-\frac{v}{E} \dot{\sigma}_{k k} \delta_{i j}$,

where $E$ and $v$ are the isotropic Young's modulus and Poisson's ratio. Notice that this form of the 
constitutive law allows for a well-defined region of elastic response which is required for the small scale yielding approximation to be used.

Here we have neglected the effects of viscoelasticity. Fracture of viscoelastic solids has been investigated by many authors, see Bradley et al. (1998) for a review. In order to reduce the number of parameters of the model and concentrate on rate effects, we have also neglected softening after initial yield and rehardening after molecular chain alignment that occurs in many polymer materials. Recently, Estevez et al. (1999) have studied crack growth with an EPZ model in glassy polymers with a constitutive law that allows for both softening and rehardening.

\section{Fracture process constitutive law}

The rate dependence for the fracture process zone is taken to follow a similar functional form as that for the bulk solid. In order to facilitate the finite element calculations it is assumed that there is always an initial linear portion in the tractionseparation law. Hence, the crack opening displacement, $\delta$, is the sum of an elastic (linear) and a plastic part. The cohesive traction obeys

$\dot{t}=\hat{\sigma} \lambda_{1} \frac{\dot{\delta}-\dot{\delta}^{\mathrm{p}}}{\delta_{\mathrm{c}}}$,

where $\lambda_{1}$ is a shape parameter of the static traction-opening law and $\delta_{\mathrm{c}}$ is the critical crack opening where tractions drop to zero. The plastic opening rate, $\dot{\delta}^{\text {p }}$, is described by

$\left(1+\frac{\dot{\delta}^{\mathrm{p}}}{\dot{\delta}_{\mathrm{o}}}\right)=\left(\frac{t}{t_{0}\left(\delta^{\mathrm{p}}\right)}\right)^{q} \quad$ for $t>t_{0}\left(\delta^{\mathrm{p}}\right)$,

$\dot{\delta}^{\mathrm{p}}=0 \quad$ for $t<t_{0}\left(\delta^{\mathrm{p}}\right)$,

$t=0 \quad$ if $\delta>\delta_{\mathrm{c}}$,

where $\dot{\delta}_{\mathrm{o}}$ is a characteristic crack opening rate, $t$ the normal traction acting on the crack plane, $q$ the rate exponent of the fracture process zone and $t_{0}\left(\delta^{\mathrm{p}}\right)$ represents the static form of the tractionseparation law. We also impose condition (3.4) requiring that the total crack opening, $\delta$, must always be less than the critical crack opening, $\delta_{\mathrm{c}}$, for all applied opening rates otherwise the traction must drop to zero. This is a somewhat arbitrary assumption of the model, which is not necessarily valid for all failure mechanisms; however it does appear to be valid for a crazing failure mechanism (Doll, 1983; Kramer and Berger, 1990). This form of the cohesive zone law is similar to that used by Freund and Lee (1990) to study dynamic viscoplastic fracture within the context of a strip yield model. In their work, to allow for an analytical solution, $t_{0}\left(\delta^{\mathrm{p}}\right)$ is constant and the rate exponent $q$ is equal to 1 .

For static loading the traction-separation law is taken to be the same as that used by Tvergaard and Hutchinson (1992). The traction-separation law is shown in Fig. 2 for a range of applied opening rates. The quantity $t_{0}$ plus the additional linear part of the traction-separation relation is represented by the curve in Fig. 2 corresponding to $\dot{\delta}=0$. The static work of separation per unit area is

$\Gamma_{0}=\int_{0}^{\delta_{\mathrm{c}}} t \mathrm{~d} \delta=\frac{1}{2} \hat{\sigma} \delta_{\mathrm{c}}\left(1+\lambda_{2}-\lambda_{1}\right)$,

where $\lambda_{1}$ and $\lambda_{2}$ are dimensionless shape parameters denoting relative opening displacements where the static traction-separation law changes slope abruptly as shown in Fig. 2.

The traction-separation law is then specified by the static intrinsic toughness $\Gamma_{0}$, peak stress $\hat{\sigma}$,

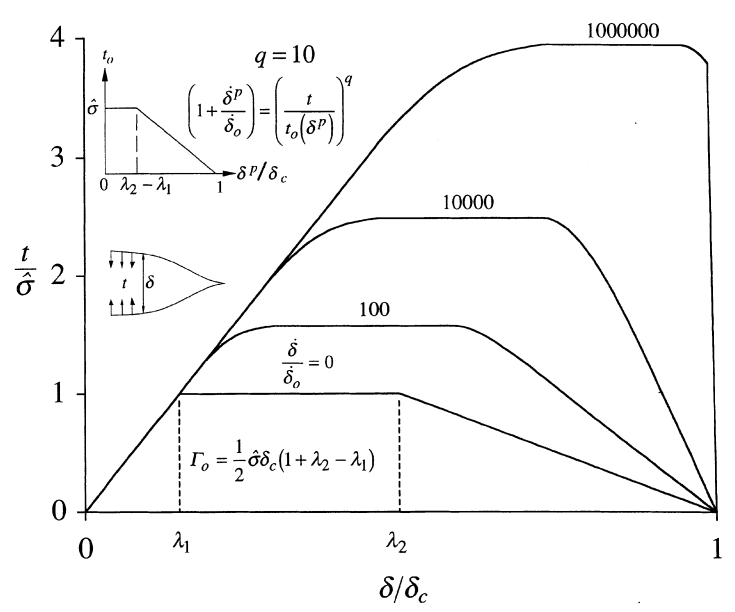

Fig. 2. Traction-separation law corresponding to various opening rates for a rate exponent, $q=10$. 
shape parameters $\lambda_{1}$ and $\lambda_{2}$, and rate properties $\dot{\delta}_{\text {o }}$ and $q$. In the context of conventional rate independent plasticity, Tvergaard and Hutchinson (1992) showed that the macroscopic toughness of the material has only a weak dependence on the shape parameters $\lambda_{1}$ and $\lambda_{2}$. We note that the determination of $\dot{\delta}_{\mathrm{o}}$ and $q$ likely remains in the realm of micromechanics and in this work we will resort to a parametric study to map out the effects of these variables.

\section{Steady-state formulation}

A quasi-static, asymptotic Mode I, plane strain analysis is carried out for a semi-infinite crack propagating at constant velocity $\dot{a}$. A small scale yielding formulation is used such that the elastic Mode I stress and displacement fields are applied far from the crack tip. Assuming that the crack is growing in the $x_{1}$ direction any rate quantity can be related to the $x_{1}$ spatial derivative through the crack velocity. For example the plastic strain rates are

$\dot{\varepsilon}_{i j}^{\mathrm{p}}=-\dot{a} \frac{\partial \varepsilon_{i j}^{\mathrm{p}}}{\partial x_{1}}$

and any other quantity can be substituted for $\varepsilon_{i j}^{\mathrm{p}}$ in (4.1) with equal validity. The iterative finite element solution procedure used to solve the steadystate problem is the same as that used by Dean and Hutchinson (1980), Parks et al. (1981), Lam and McMeeking (1984), Wei and Hutchinson (1997a,b), and Dhirendra and Narasimhan (1998).

The virtual work statement for the quasi-static steady-state problem is

$$
\begin{aligned}
\int_{V} \delta \varepsilon_{i j} C_{i j k l} \varepsilon_{k l} \mathrm{~d} V= & \int_{S} \delta u_{i} T_{i} \mathrm{~d} S \\
& +\int_{V} \delta \varepsilon_{i j} C_{i j k l} \varepsilon_{k l}^{\mathrm{p}} \mathrm{d} V,
\end{aligned}
$$

where $S$ is the boundary of the volume $V, u_{i}$ are the displacements, $\varepsilon_{i j}$ is the infinitesimal strain tensor, $C_{i j k l}$ the tensor of isotropic elastic stiffness, $T_{i}$ the traction acting on the boundary and $\varepsilon_{i j}^{\mathrm{p}}$ are the plastic strains. Given a solution for the displacement field, the plastic strains can be obtained by integrating the plasticity law along streamlines. In this work, a backward Euler method as described in the ABAQUS Theory Manual (1997) is used to integrate the constitutive law. The new distribution of plastic strains is then integrated as a body force in the second term of the right-hand side of (4.2) and a new solution for the displacement field is computed. The procedure is repeated until convergence is achieved.

\section{Results}

In this section, results for the macroscopic steady-state toughness will be presented as a function of model parameters. The condition imposed on the finite element calculations for steadystate crack propagation is that $\delta=\delta_{\mathrm{c}}$ at $x_{1}=0$. Dimensional analysis suggest that the macroscopic steady-state toughness depends on the following dimensionless parameters:

$\frac{\Gamma_{\mathrm{ss}}}{\Gamma_{0}}=\bar{\Gamma}\left(\frac{\dot{a}}{R_{0} \dot{\varepsilon}_{0}}, \frac{\hat{\sigma}}{\sigma_{y}}, \frac{\dot{\delta}_{\mathrm{o}}}{\delta_{\mathrm{c}} \dot{\varepsilon}_{0}}, m, q, \frac{\sigma_{y}}{E}, v, \lambda_{1}, \lambda_{2}\right)$,

where

$R_{0}=\frac{1}{3 \pi}\left(\frac{E}{1-v^{2}}\right) \frac{\Gamma_{0}}{\sigma_{y}^{2}}=\frac{1}{3 \pi}\left(\frac{K_{0}}{\sigma_{y}}\right)^{2}$

is the approximate size of the plastic zone when the applied stress intensity, $K$, is equal to $K_{0}$, where $K_{0}$ is related to $\Gamma_{0}$ through (5.2). Previous studies on rate independent materials have demonstrated that the last four parameters in (5.1) are of secondary importance. For the bulk of this study we will use the following values for these parameters: $E / \sigma_{y}=50, v=0.35, \lambda_{1}=0.15$ and $\lambda_{2}=0.5$. This leaves us with the task of mapping out the effects of the remaining five parameters. However, in Section 5.3 we will examine the crack plane traction and opening profiles using different shapes of the cohesive zone law.

\subsection{Rate independent fracture process zone}

The first set of results shown in Fig. 3 are for a solid with rate exponent $m=10$ and a rate independent fracture process zone. The rate 


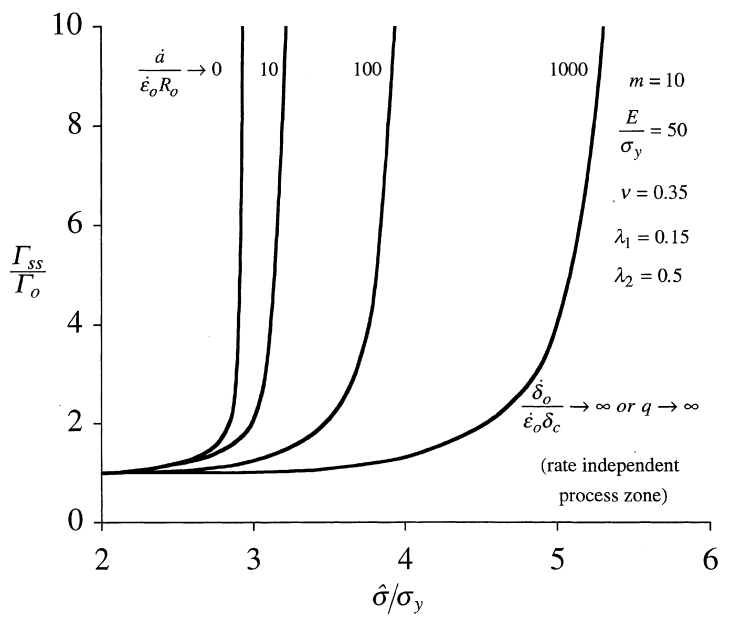

Fig. 3. The steady-state toughness as a function of the peak stress for different crack velocities with a rate independent fracture process.

independent fracture process zone is a limiting case of Eqs. (3.1)-(3.4) with $\dot{\delta}_{\mathrm{o}} /\left(\dot{\varepsilon}_{0} \delta_{\mathrm{c}}\right) \rightarrow \infty$ or $q \rightarrow \infty$. Justification for a rate independent process zone associated with a rate dependent solid will be given in Section 6. Fig. 3 plots the steady-state toughness as a function of the peak cohesive stress in the fracture process zone. For values of $\hat{\sigma}<2 \sigma_{y}$ the plasticity in the bulk solid is not of sufficient intensity to induce a significant amount of dissipation and the steady-state toughness is only slightly greater than $\Gamma_{0}$. For exceedingly slow crack velocities the model reduces to a rate-independent elastic-perfectly plastic material where the solid cannot sustain normal tractions greater than approximately $2.96 \sigma_{y}$ ahead of the crack tip. Hence, if $\hat{\sigma}>2.96 \sigma_{y}$, then the fracture process zone cannot separate and $\Gamma_{\mathrm{ss}}$ is infinite. At finite crack velocities the strain rate effects in the solid become relevant. Increasing crack velocities allow for elevated normal traction acting on the crack plane. However, the normal traction ahead of the crack tip cannot exceed $\hat{\sigma}$ which limits the intensity of the plastic deformation and hence the toughness of the material decreases as crack velocity increases. The effects shown in Fig. 3 are analogous to strain hardening or strain gradient hardening effects in rate independent materials (Wei and Hutchinson, 1997a,b). The general trend is that material hard-

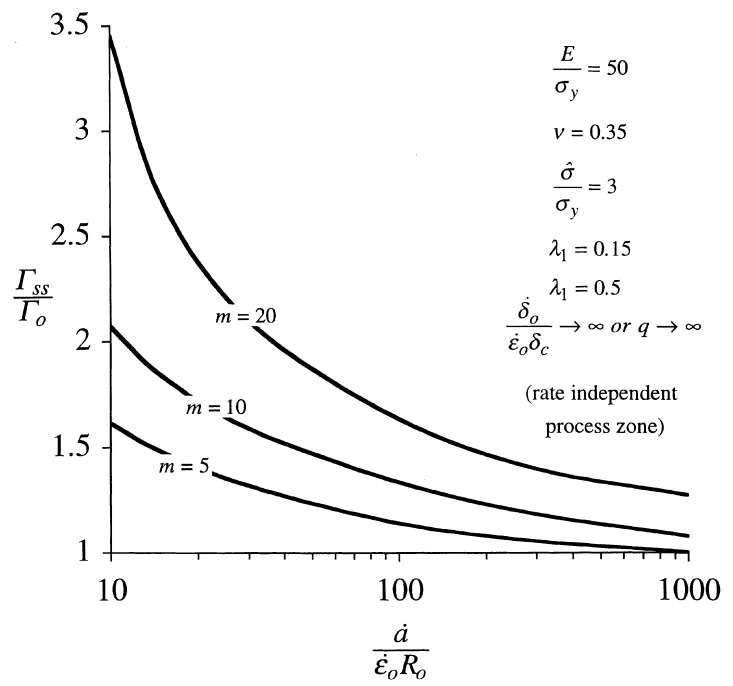

Fig. 4. Influence of the rate exponent of the bulk material on the steady-state toughness as a function of the crack velocity for a rate independent fracture process.

ening elevates tractions ahead of the crack tip, which promote the separation of the fracture process zone.

Fig. 4 further illustrates the effects of crack velocity on $\Gamma_{\mathrm{ss}}$ for the rate independent fracture process zone. Three curves are shown in Fig. 4 each for a different level of the strain rate exponent of the bulk solid and a peak cohesive stress of $\hat{\sigma}=3 \sigma_{y}$. Again, $\Gamma_{\text {ss }}$ decreases as the crack velocity increases. Lower values of $m$ magnify the solids tendency for hardening due to rate effects, and therefore at a given crack velocity, the toughness of a material with a low value of $m$ is less than that of a material with a large strain rate exponent. Overall, the trends in $\Gamma_{\mathrm{ss}}$ as a function of $\dot{a}$ or $m$ for a material with a rate-independent fracture process zone are readily explained by considering the hardening ability of the bulk solid.

\subsection{Rate dependent fracture process zone}

The introduction of a rate dependent fracture process zone sets up a competition between the hardening of the solid and the strengthening of the cohesive zone. As such, trends in the steady-state toughness are not as straightforward as that for the rate independent fracture process. Further- 
more, $\Gamma_{\mathrm{ss}}$ is sensitive to the two rate parameters associated with the fracture process, $q$ and $\dot{\delta}_{\mathrm{o}}$. It is likely that $q$ and $\dot{\delta}_{\text {o }}$ will be difficult to measure directly, leaving their determination to micromechanical models of the fracture process.

In the limit as $\hat{\sigma} / \sigma_{y} \rightarrow 0$ the steady-state toughness is equal to the static work of fracture. At first glance this is not obvious when the fracture process zone is rate dependent. The limit of $\hat{\sigma} / \sigma_{y} \rightarrow 0$ corresponds to two elastic half spaces bonded by a cohesive zone described by Eqs. (3.1)(3.4). Recall that for a simple Dugdale zone the cohesive zone length scales as $E \delta_{\mathrm{c}} / \hat{\sigma}$ with the opening rate scaling as $\dot{a} \hat{\sigma} / E$ which approaches zero as $\hat{\sigma} \rightarrow 0$. Hence, the static traction-separation law is valid as $\hat{\sigma} / \sigma_{y} \rightarrow 0$ and the macroscopic work of fracture is $\Gamma_{0}$ since any plastic dissipation is also negligible in this limit. For the case where $q=1$ where tractions are considerably more sensitive to opening rates than any cases we consider, Freund and Lee (1990) also obtain this result.

Fig. 5 plots $\Gamma_{\text {ss }}$ as a function of $\hat{\sigma}$ for various crack velocities. The rate exponent for the fracture process was taken to be equal to that of the solid for these simulations, $q=m=10$. Focusing first on the solid curves with the normalized reference

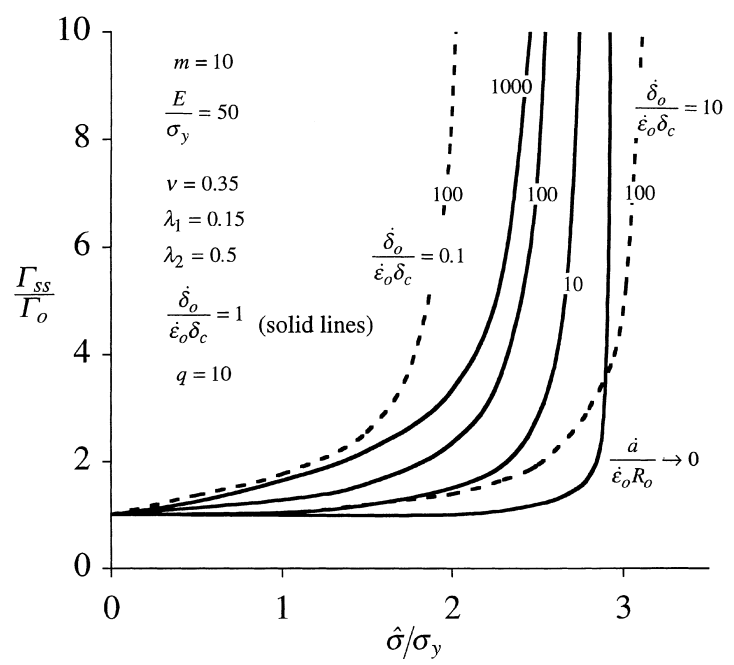

Fig. 5. The steady-state toughness as a function of the peak stress for different crack velocities with a rate dependent fracture process characterized by $q=10$. The dashed curves illustrate the effect of the reference opening rate on the steady-state toughness. crack opening rate $\dot{\delta}_{\mathrm{o}} /\left(\dot{\varepsilon}_{0} \delta_{\mathrm{c}}\right)=1$, we note that steady-state toughness now increases as the crack velocity increases. Within the range of $\Gamma_{\mathrm{ss}}$ shown, the strengthening of the fracture process dominates the hardening in the bulk solid. The two dotted lines in Fig. 5 are the results for a normalized crack velocity of $\dot{a} /\left(\dot{\varepsilon}_{0} R_{0}\right)=100$ and reference crack opening rates of $\dot{\delta}_{\mathrm{o}} /\left(\dot{\varepsilon}_{0} \delta_{\mathrm{c}}\right)=0.1$ and 10. For a given crack opening rate, Eq. (3.2) indicates that the traction acting across the crack faces decreases as $\dot{\delta}_{\mathrm{o}}$ increases. Crudely, increasing $\dot{\delta}_{\text {o }}$ is similar to decreasing $\hat{\sigma}$ at a given opening rate.

Notice that the solid curves in Fig. 5 seem to approach the $\dot{a} /\left(\dot{\varepsilon}_{0} R_{0}\right) \rightarrow 0$ curve from the left as the crack velocity decreases. This appearance is due to the range of $\Gamma_{\mathrm{ss}}$ shown in the figure. Notice that the $\dot{a} /\left(\dot{\varepsilon}_{0} R_{0}\right)=100, \dot{\delta}_{\mathrm{o}} /\left(\dot{\varepsilon}_{0} \delta_{\mathrm{c}}\right)=10$ simulations cross over the $\dot{a} /\left(\dot{\varepsilon}_{0} R_{0}\right) \rightarrow 0$ curve. This is also the case for the $\dot{\delta}_{\mathrm{o}} /\left(\dot{\varepsilon}_{0} \delta_{\mathrm{c}}\right)=1$ cases; however the crossover occurs at much higher values of $\Gamma_{\mathrm{ss}}$. A similar change in the toughness vs crack velocity trend is evident for a value of $q=2 m$ to be presented next.

As we have stated throughout $q$ and $\dot{\delta}_{\text {o }}$ could be obtained from a micromechanical analysis. Kramer and Berger (1990) present a model for craze widening and under their assumptions the rate exponent for the craze widening is predicted to be twice that for bulk deformations. We note that their assumed form of the bulk constitutive law was power law viscous which is similar to Eq. (2.1) except that no yield surface exists, i.e. the additive constant of 1 is removed from the left-hand side of (2.1) and plastic straining occurs at all stress levels. For increasing levels of $q$, Eq. (3.2) indicates that for a given applied opening rate the traction acting across the crack surfaces decreases. As shown in Figs. 3 and 4 the rate independent fracture process limit corresponds to $q \rightarrow \infty$. Hence, an increase in $q$ will have similar effects on $\Gamma_{\mathrm{ss}}$ as an increase in $\dot{\delta}_{\text {o }}$. Fig. 6 plots $\Gamma_{\text {ss }}$ as a function of $\hat{\sigma}$ for three crack velocities and $q=2 m=20$. Here again a crossover in the trend of toughness vs velocity occurs. For static peak cohesive stresses lower than approximately $2.7 \sigma_{y}, \Gamma_{\mathrm{ss}}$ increases slightly as $\dot{a}$ increases. However, for $\hat{\sigma}>2.7 \sigma_{y}$ the toughness decreases dramatically as the crack velocity 


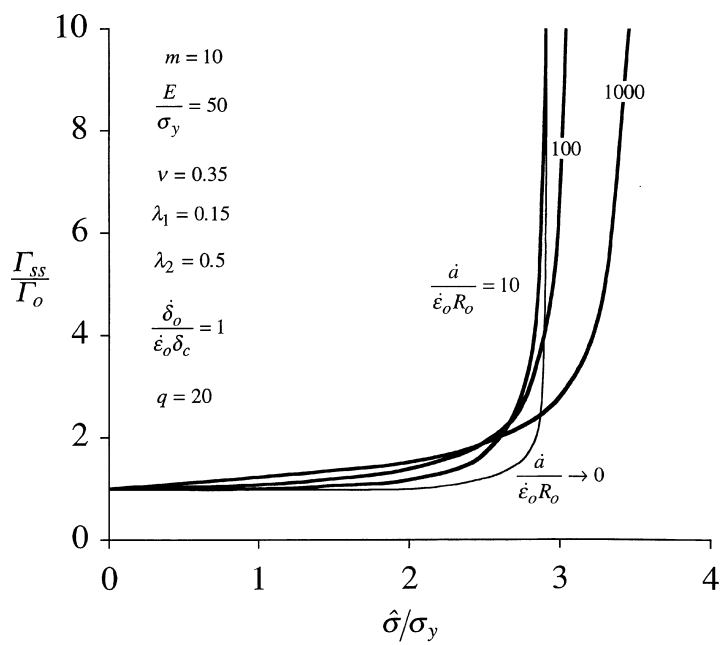

Fig. 6. The steady-state toughness as a function of the peak stress for different crack velocities with a rate dependent process zone characterized by $q=20$.

increases (within the range of velocities shown). This transition in behavior is illustrated again in Fig. 7 with $\Gamma_{\text {ss }}$ plotted against $\dot{a}$ for 4 levels of $\hat{\sigma}$. Notice that for $\hat{\sigma} \approx 2.7 \sigma_{y}$ the toughness would essentially be independent of the crack velocity in the range of $\dot{a} /\left(\dot{\varepsilon}_{0} R_{0}\right)=10-1000$; an interesting

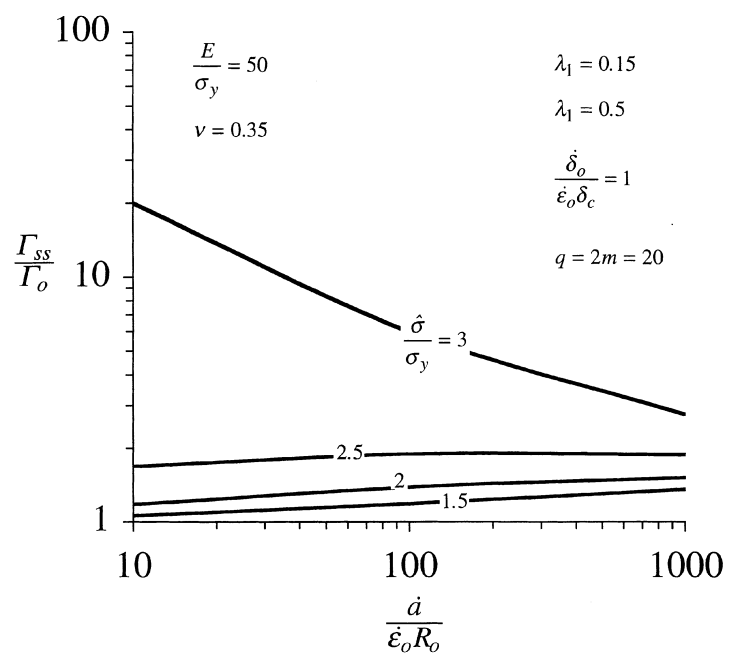

Fig. 7. Influence of the peak stress on the steady-state toughness as a function of the crack velocity for a rate dependent fracture process characterized by $q=20$. result for a system with rate dependence in both the solid and the fracture process.

\subsection{Crack plane traction and opening}

To elucidate the features of the fracture process we now present results for the traction and opening profiles ahead of the crack tip from simulations with different rate independent forms of the traction-separation law. The generalization to rate dependent behavior still follows Eqs. (3.1)-(3.4). The results plotted in Figs. $8-10$ are for simulations with $\hat{\sigma}=3 \sigma_{y}, \dot{a} /\left(\dot{\varepsilon}_{0} R_{0}\right)=100, q=2 m=20$ and $\dot{\delta}_{\mathrm{o}} /\left(\dot{\varepsilon}_{0} \delta_{\mathrm{c}}\right)=1$; however the general trends of the solution variables to be presented are similar for other sets of model parameters as well. We note that the $\Gamma_{\text {ss }}$ values predicted from the two different shapes of the traction-separation law differed by less than $1 \%$. Also the size of the actual plastic zone, $R_{\mathrm{p}}$, is used to normalize the distance from the crack tip and is defined as

$R_{\mathrm{p}}=R_{0} \frac{\Gamma_{\mathrm{ss}}}{\Gamma_{0}}$

Fig. 8 plots the crack opening displacement (left ordinate) and the normal stress acting on the cohesive surfaces (right ordinate) as a function of the distance ahead of the crack tip. The solid lines (Case 1) are results from the simulation with the

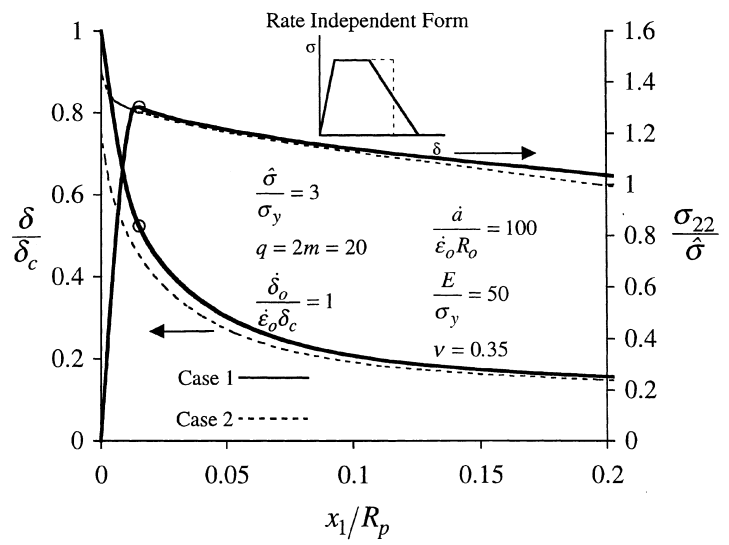

Fig. 8. Crack opening displacement and cohesive traction ahead of the crack tip for two different shapes of the cohesive zone law. The rate dependent fracture process is characterized by $q=20$. 


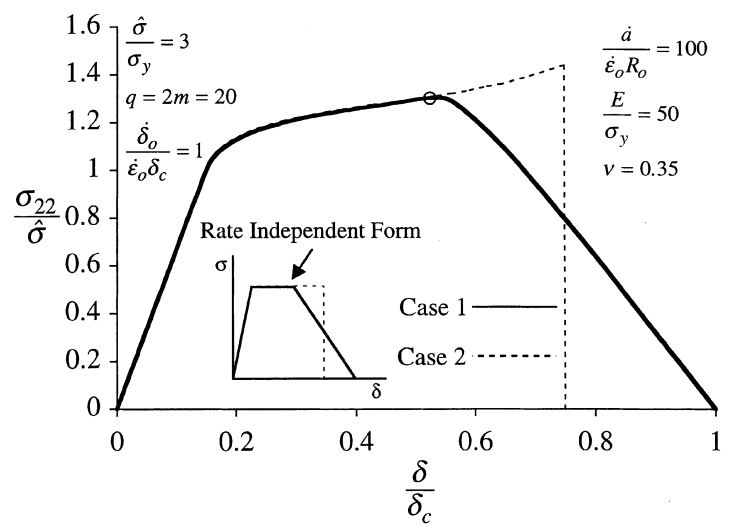

Fig. 9. The cohesive traction-separation profile that each material point experiences as the crack passes for two different shapes of the cohesive zone law.

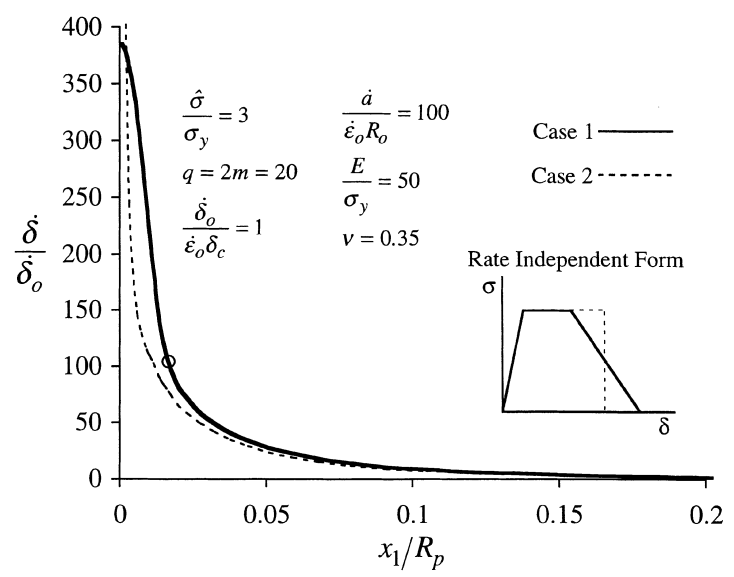

Fig. 10. Crack opening rate ahead of the crack tip for two different shapes of the cohesive zone law.

shape of the traction-separation law used to generate the previous results, i.e. $\lambda_{1}=0.15$ and $\lambda_{2}=0.5$. The dashed lines (Case 2) are from a simulation with a traction-separation law with the same rate independent peak stress and fracture energy as for Case 1 but with a precipitous drop in cohesive stress at the critical opening as drawn in Figs. $8-10$, i.e. $\lambda_{1}=0.2$ and $\lambda_{2}=1$. Note that for the purpose of comparison $\delta_{\mathrm{c}}$ always refers to the critical opening for the cohesive zone law of Case 1 and consequently the critical opening displacement for Case 2 is $0.75 \delta_{\mathrm{c}}$.
The crack opening profiles for both tractionseparation laws are similar. As shown in Fig. 8 the crack opening displacement decreases monotonically with increasing distance from the crack tip. The contrast near the crack tip in the crack face traction profiles occurs due to the difference in the continuity of the traction-opening law at final separation. For Case 1, the traction goes to zero in a continuous fashion as the opening approaches the critical opening; hence the traction at $x=0$ must vanish and build to a maximum at some distance from the crack tip. In comparison, the traction-separation law is discontinuous in Case 2 and the traction to the right of the crack tip will not be zero, and in fact attains a maximum value. The open circle on the traction profile of Case 1 indicates the position where the maximum traction occurs.

Fig. 9 displays the traction vs separation that every material point on the cohesive surface experiences as the crack tip passes. The area under these curves represents the intrinsic contribution to the macroscopic toughness of the material. The energies required to separate the process zones in Cases 1 and 2 are $1.3 \Gamma_{0}$ and $1.24 \Gamma_{0}$, respectively. Recall that the steady-state toughness of the materials in Cases 1 and 2 differed by less than $1 \%$, $\Gamma_{\mathrm{ss}} \approx 5.8 \Gamma_{0}$ in both cases. Hence, the plastic dissipation must be slightly greater in Case 2 to account for the difference fracture process energy. Furthermore, Figs. 8 and 9 demonstrate that differences between the two cases occur only very near the crack tip during the final separation of the process zone.

Lastly, Fig. 10 is a plot of the crack opening rate as a function of the distance ahead of the crack tip. First, notice the location of the open circle for Case 1. This position corresponds to the position where the maximum traction occurs as indicated in Fig. 8. Consider a material point on the crack plane. Far downstream the traction, opening and opening rate are all equal to zero and as the crack tip approaches, these variables increase gradually. Close to the crack tip the traction at a material point reaches a maximum and then diminishes (Case 1) or increases monotonically (Case 2) and the crack opening rate increases dramatically. This region of the opening 
rate profile is dependent on the form of the traction-separation profile as demonstrated by the differences between Cases 1 and 2. Notice that the opening rate at the crack tip for Case 1 appears to approach a finite value, while the results for Case 2 suggest a singularity in the opening rate. The finite element solution cannot confirm a singularity in the opening rate for Case 2. However, it is well known that the analytical result for a steadily growing crack between two elastic half spaces bonded by a Dugdale zone, which has a similar abrupt drop in traction at the critical opening displacement as for Case 2, yields a logarithmic singularity in the opening rate at the crack tip.

\section{The fracture process for rubber-toughened epox- ies}

In this section, we will connect the EPZ model of the previous sections with micromechanical void cell computations in order to interpret the experimental observations of Du et al. (2000) on the toughness of a rubber modified epoxy. As such, the experimental findings of Du et al. (2000) are presented first. Then, utilizing the analysis from the steady-state fracture model, qualitative predictions about the features of the fracture process in this material are produced. Finally, void cell computations at different mesoscopic applied strain rates are performed assuming that a critical stress fracture criterion in the intervoid ligament governs final failure. The critical stress fracture criterion in the ligament is motivated by observations of the fracture planes from the experiments. This process of coupling the EPZ model with the void cell calculations and experimental observations reveals the significant features of the fracture process for this material.

\subsection{Macroscopic observations and deductions of the model}

Experimental observations of the effects of crack velocity on the toughness of rubber-toughened epoxy were recently performed by $\mathrm{Du}$ et al. (2000). The rate dependent fracture behavior of a rubber modified epoxy (10\% volume fraction of $1-$ $2 \mu \mathrm{m}$ diameter rubber particles) was investigated using double cantilever beam tests at various load point displacement rates. Fig. 11 plots the fracture toughness as a function of crack advance for three different applied displacement rates. During these tests, the crack velocity first increases slowly and, near the peak toughness, dramatically accelerates with crack advance becoming unstable. Hence, the interpretation of the crack resistance curves is not straightforward due to transient effects associated with the changing crack velocity, i.e. no steadystate fracture toughness can be inferred from these experiments. Nonetheless, a clear decrease of the fracture resistance, $\Gamma_{\mathrm{R}}$, with increasing crack speed is observed. The crack velocity of the fastest test is typically three times larger than in the slowest test at the same level of crack advance $\Delta a$. The fracture toughness at crack initiation is about $2 \mathrm{~kJ} / \mathrm{m}^{2}$ and changes only slightly with increasing crack velocity. As discussed by Du et al. (1998), this value is an upper bound on the true initiation toughness due to the experimental difficulty in determining the onset of crack propagation. Assuming that the initiation toughness does not change significantly, the trend of Fig. 11 suggests that for a test performed at larger displacement rates (crack velocities) little to no increase of toughness would be observed during propagation.

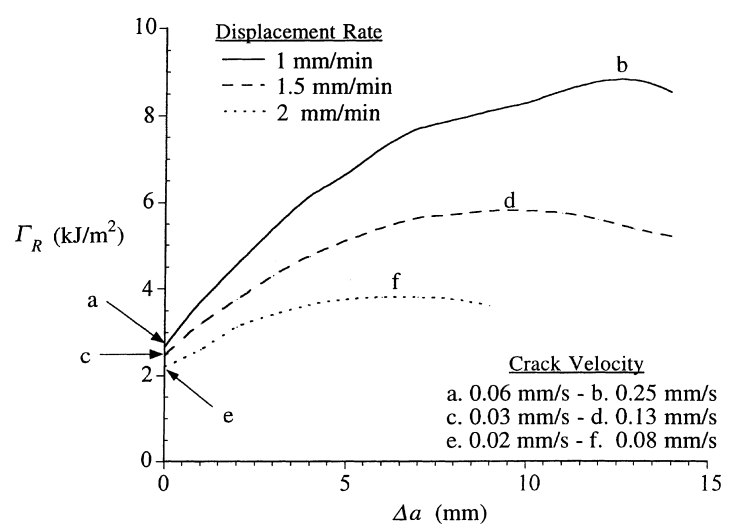

Fig. 11. Experimental crack growth resistance curves for a modified rubber epoxy. The tests were performed by $\mathrm{Du}$ et al. (2000) on double cantilever beam specimen loaded at three different displacement rates. The crack tip velocities at initiation and just prior to instability are recorded on the plot. 
From these fracture toughness measurements alone we can infer some qualitative characteristics of the fracture process by considering the results from the EPZ model presented in Figs. 3, 5 and 6. First, the macroscopic toughness of the material decreases as the crack velocity increases implying that the rate hardening effects in the bulk dominate the rate strengthening effects in the fracture process zone. Figs. 3 and 6 show that decreases in toughness with increasing crack velocity occur in the regime where the rate independent peak cohesive stress, $\hat{\sigma}$, is greater than about three times the rate independent yield stress of the bulk solid. Furthermore, in the regime where $\hat{\sigma}$ is approximately $3 \sigma_{y}$ the rate properties of the fracture process zone, $q$ and $\dot{\delta}_{\mathrm{o}}$, significantly effect the sensitivity of the macroscopic toughness to the crack velocity. Focusing on the effects of $q$, comparison of Figs. 3 and 6 illustrates that the macroscopic toughness increases more rapidly for smaller values of $q$ (recall that the rate independent fracture process used to generate Fig. 3 is the limit as $q \rightarrow \infty$ ). The experimental observations suggest that for a threefold increase in crack velocity the macroscopic toughness of the material is decreased by about a factor of 2 . Therefore, it is reasonable to believe that the fracture process for this material is less rate sensitive than the bulk material, i.e. $q>m$. To demonstrate how this is possible, we now resort to micromechanical calculations of void growth and ligament fracture around the rubber particles.

\subsection{Microscopic observations and a void cell model}

Along with macroscopic measurements of the fracture toughness, Du et al. (2000) also observed the morphology of the fracture surfaces of the broken specimens. The fracture surfaces consist of dimples separated by flat, seemingly brittle ligament failures. Similar observations have been reported in the literature by Pearson and Yee (1986), Kinloch et al. (1986) and Pearson and Yee (1991). A critical porosity of $17 \%$ has been estimated from the size of the dimples. This porosity is constant along the part of the crack front corresponding to the stable crack growth regime, which encompasses almost two orders of magnitude of crack velocity. On the part of the fracture surface corresponding to unstable fast cracking the critical porosity drops to $10.5 \%$, implying almost no void growth, see also Pearson and Yee (1986). The increase in porosity from $10 \%$ to $17 \%$ requires substantial plastic dissipation due to void growth in the material. From these observations we propose a simple model for the fracture process. During void growth the ligaments between voids are plastically deformed to large strains causing an increase of the local stress from orientational hardening induced by the alignment of the polymer chains. Large stresses can then accrue in the ligament allowing failure by a brittle mechanism. Hence, we assume that the cavities will grow until the stress state between the voids attains a critical level leading to final fracture of the remaining ligament.

Void cell computations with typical epoxy properties were performed at different levels of applied overall strain-rate. The system that is analyzed in the present work is envisioned as a periodic array of hexagonal prism unit cells each containing a centered, initially spherical void. For the sake of simplicity, this assemblage is approximated by circular cylinders allowing for axisymmetric calculations, which have been shown to provide a good approximation to the hexagonal cells (e.g. Worswick and Pick, 1990). The mesoscopic principal strains and a special "effective" strain measure are given by

$$
\begin{aligned}
& E_{r}=\ln \left(\frac{L_{r}}{L_{r 0}}\right), \quad E_{z}=\ln \left(\frac{L_{z}}{L_{z 0}}\right), \\
& E_{\text {eff }}=\frac{2}{3}\left|E_{z}-E_{r}\right| .
\end{aligned}
$$

The mesoscopic true principal stresses, $\Sigma_{r}$ and $\Sigma_{z}$, are the average forces at the cell boundary per current area. The effective stress, hydrostatic stress, and stress triaxiality measures are

$$
\begin{aligned}
& \Sigma_{\mathrm{e}}=\left|\Sigma_{z}-\Sigma_{r}\right|, \quad \Sigma_{\mathrm{h}}=\frac{1}{3}\left(\Sigma_{z}+2 \Sigma_{r}\right), \\
& T=\frac{\Sigma_{\mathrm{h}}}{\Sigma_{\mathrm{e}}} .
\end{aligned}
$$


One difficulty in determining a simple one-dimensional process zone model from a micromechanical analysis is that the stress state representative of the loading history experienced by a material element deforming near the crack tip must be assumed. In metals, Anderson (1977) and Tvergaard and Hutchinson (1992) have argued that uniaxial straining is a good approximation to the crack tip deformation state since after the maximum stress is reached, damage localizes in one band while the rest of the material unloads, behaving as a rigid body. However, for rubbertoughened epoxy, the relatively large porosities relieve the triaxial stress field (see also Yee et al. 1993). Miao and Drugan (1995) analyzed the problem of steady-state crack propagation in a porous elastic-perfectly plastic matrix, using the Gurson model (Gurson, 1977). Specifically, they showed results for the effect of the porosity on the stress triaxiality at the tip of a growing crack. The stress triaxiality decreases almost linearly with porosity in the analyzed range of porosity (from 0 to $5 \%$ ). Extrapolation of their results to the porosity of interest in this paper, i.e. $10 \%$, gives stress triaxiality equal to 0.8 . We note that accurate determination of the stress triaxiality is compromised by both the extrapolation procedure and by the fact that the Gurson model loses accuracy at high levels of porosity. However, the approximations used are within reason and $T=0.8$ has been chosen for the computations presented herein.

The void cell computations were performed within the finite strain setting using the generalpurpose finite element program ABAQUS version 5.7 (1997). Axisymmetric 9-node isoparametric elements with reduced integration were implemented. We assume that the rubber particles debond or cavitate at low strains when the epoxy is deforming elastically as suggested by the analysis of Lazzeri and Bucknall (1995) and therefore the particle is treated as a void. All the computations have been performed for an initial porosity of $10 \%$. The void is initially spherical and the void spacing is the same in the $r$-and $z$-directions.

As in the first part of this paper, isotropic elasticity is assumed as well as a strain-rate dependent plasticity model

$$
\left(1+\frac{\dot{\bar{\varepsilon}}^{\mathrm{p}}}{\dot{\varepsilon}_{0}}\right)=\left(\frac{\bar{\sigma}}{\sigma_{0}}\right)^{m} \quad \text { if } \bar{\sigma} \geqslant \sigma_{0},
$$

$\dot{\bar{\varepsilon}}^{\mathrm{p}}=0 \quad$ if $\bar{\sigma}<\sigma_{0}$.

In an attempt to account for orientational hardening, isotropic linear hardening is implemented above a critical effective plastic strain $\varepsilon_{\mathrm{c}}$. Then the reference stress $\sigma_{0}$ is defined as

$\sigma_{0}=\sigma_{y} \quad$ if $\bar{\varepsilon}^{\mathrm{p}} \leqslant \varepsilon_{\mathrm{c}}$,

$\sigma_{0}=\sigma_{y}+H\left(\bar{\varepsilon}^{\mathrm{p}}-\varepsilon_{\mathrm{c}}\right) \quad$ if $\bar{\varepsilon}^{\mathrm{p}}>\varepsilon_{\mathrm{c}}$.

This description of orientational hardening is approximate for two reasons. First, orientational hardening is most effectively modeled with kinematic hardening and requires the introduction of a back stress tensor (Boyce et al., 1988; Wu and Van der Giessen, 1993). However, for nearly proportional loading, as analyzed in the cell computations, no difference exists between kinematic and isotropic hardening. Second, a geometrical model for the onset of the orientational hardening should be based on a critical principal plastic strain rather than a critical effective plastic strain. However, as only one stress state characterized by a constant stress triaxiality is addressed, this approximation will only have a minor effect on the analysis presented here.

Most of the computations have been performed with constant applied axial displacement rate. We have also explored how the cell reacts when a more realistic displacement rate history is applied typical of that experienced by a material element as the crack tip approaches. More precisely, the displacement rate history was taken from the results generated from the steady-state solution with the fracture process zone from Case 2 of Section 5.3.

Table 1 summarizes the values of the parameters used to generate the results presented in this section. These mechanical parameters come from experimental measurements of Sener (1999) on an epoxy resin strained at different rates which agree very well with values given by Crocombe et al. (1995) or used by Huang and Kinloch (1992). The overall stress and strain quantities as well as the current porosity and the stresses in the intervoid 
Table 1

\begin{tabular}{llllllll}
\hline$f_{0}$ & $E / \sigma_{y}$ & $v$ & $\varepsilon_{\mathrm{c}}$ & $E / \sigma_{y}$ & $m$ & $\dot{\bar{E}} / \dot{\varepsilon}_{0}$ & Stress state \\
\hline 0.1 & 50 & 0.35 & 0.3 & 7.5 & 10 & $10^{-4}-10^{6}$ & $T=0.8$ \\
\hline
\end{tabular}

ligament are computed. The maximum value of the mean stress in the ligament will be considered as the fracture criterion for final ligament failure.

\subsection{Results}

Fig. 12 plots the variation of the normalized overall axial stress $\Sigma_{z} / \sigma_{y}$ as a function of the normalized axial displacement $\delta / X_{0}$ for cell simulations performed at constant axial displacement rates $\dot{\delta} / \dot{\varepsilon}_{0} X_{0}$ with $T=0.8$. Fig. 12 also displays the result of a cell simulation performed with a varying rate corresponding to a crack velocity $\dot{a} / \dot{\varepsilon}_{0} R_{0}=100$ and other model parameters discussed for Case 2 of Section 5.3. The maximum displacements in Fig. 12 corresponds to one particular choice for the fracture criterion, $\sigma_{\mathrm{mc}} / \sigma_{y}=3.5$. A significant change in the behavior of the cell is observed when the normalized displacement rate increases from $10^{4}$ to $10^{5}$. Also, the peak stress decreases slightly with increasing applied displacement rates.

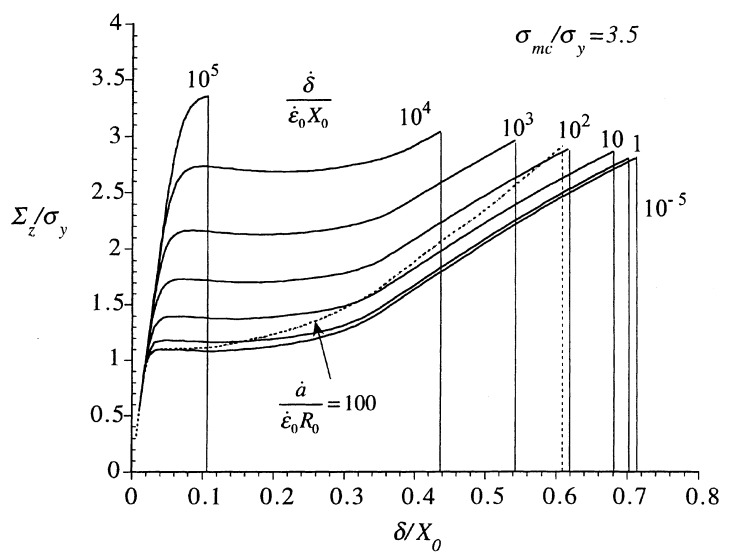

Fig. 12. Overall stress displacement curves for the void cell computations. Solid lines correspond to different levels of uniform applied displacement rates. The dotted line refers to a result obtained with a varying applied displacement rate consistent with the steady-state calculation of Case 2 in Figs. 8-10.
Fig. 13 plots the work done in straining the cell (normalized by the yield stress and by the void spacing, $\left.\Gamma_{0} / \sigma_{y} X_{0}\right)$ and the overall peak stress, $\Sigma_{z}^{\max } / \sigma_{y}$, as a function of the applied displacement rate. The transition in behavior as the rate goes from $10^{4}$ to $10^{5}$ is clear. For rates below the transition, $\Gamma_{0} / \sigma_{y} X_{0}$ is nearly constant while $\Sigma_{z}^{\max } / \sigma_{y}$ increases slightly with increasing rate. In this regime, the sensitivity of $\Sigma_{z}^{\max } / \sigma_{y}$ to $\dot{\delta} / \dot{\varepsilon}_{0} X_{0}$ has been characterized by the functional form of (3.2), giving a value of $q$ equal to 100 . The fact that the rate exponent of the peak stress is 10 times that of the bulk solid implies that, to a good approximation, the peak stress can be considered as rate independent. At first glance these two results, that the peak stress and fracture energy of the cohesive zone are independent of the applied opening rate, imply that the fracture process is rate independent. However, strictly speaking, the cohesive zone model emanating from the micromechanical analysis cannot be considered as truly rate independent. Fig. 12 illustrates that the shape of the traction-separation profile is dependent on the

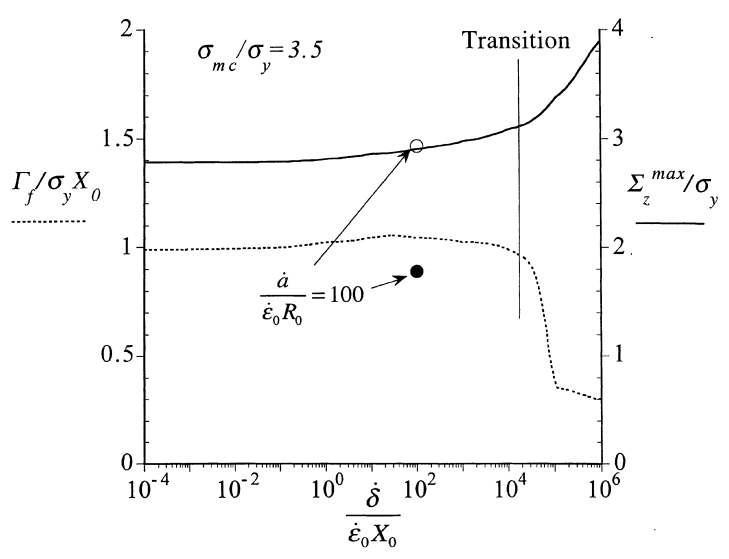

Fig. 13. Work spent per unit area and maximum overall stress obtained with the void cell computations as a function of the opening rate. 
opening rate. When a crack propagates through the material the opening rate at a material point is initially very small but increases rapidly as the crack tip approaches. Hence, this type of varying rate history yields a smaller work of separation than the uniform rate history. To illustrate this effect, the work of separation and peak stress corresponding to the crack velocity $\dot{a} / \dot{\varepsilon}_{0} R_{0}=100$ have been added in Fig. 13 using the average opening rate to plot along the abscissa. The peak stress predicted from the varying opening rate simulation is in accord with the predictions from the constant rate calculations. However, the work of fracture for the varying opening rate $\left(\dot{a} / \dot{\varepsilon}_{0} R_{0}=100\right)$ is $10 \%$ lower than the work of fracture for the uniform displacement rate simulations. It is worth noting that the traction-separation profile for a varying displacement rate corresponding to an extremely small crack velocity will coincide with the result for a very slow uniform applied displacement rate.

As a final check on the void cell calculations, the final porosity computed from the model is compared to the experimentally observed porosities. In agreement with the observations of $\mathrm{Du}$ et al. (2000) a fairly constant critical porosity is found below the peak stress/work of fracture transition $\left(f_{\mathrm{c}}=0.19\right.$ in the computations while $f_{\mathrm{c}}=0.17$ experimentally) while above the transition almost no void growth occurs. The transition observed in the calculations gives a possible explanation for the cracking instability observed when the crack velocity increase above a given value. The transition predicted by the void cell calculations supplies a credible explanation for the instability observed experimentally as the crack velocity increases. The model suggests that for sufficiently high crack velocities a large stress in the intervoid ligament can be attained before a significant amount of plasticity accumulates, resulting in a reduction of the toughness of the fracture process.

\section{Discussion and conclusions}

The results of the model with the rate independent fracture process can be divided into two regimes, $\hat{\sigma}<2.5 \sigma_{y}$ and $\hat{\sigma}>2.5 \sigma_{y}$, see Fig. 3. For values of $\hat{\sigma}$ less than about $2.5 \sigma_{y}$ rate effects in the bulk solid have little significance in determining the steady-state fracture toughness of the material. In this regime of cohesive stresses $\Gamma_{\mathrm{ss}}$ is not enhanced by extrinsic toughening effects associated with the wake of residual plastic strains. While for $\hat{\sigma}$ above $2.5 \sigma_{y}$, and more significantly above $3 \sigma_{y}$, extrinsic toughening represents a significant portion of $\Gamma_{\mathrm{ss}}$ for relatively slow crack velocities. However, due to rate hardening, high crack velocities allow the solid to overcome the peak cohesive stress of the fracture process more easily, thus reducing the steady-state toughness.

For a rate dependent fracture process zone where both the toughness and strength increase with increasing crack opening rate, predictions of the model are more complicated. However, a simple rule of thumb could be proposed: if the peak cohesive stress is less than approximately $3 \sigma_{y}$ then the steady-state toughness will increase with increasing crack velocity while the opposite trend holds for $\hat{\sigma}>3 \sigma_{y}$. The multiplicative coefficient of 3 is a remnant of the perfectly plastic description of the static stress-strain behavior and the introduction of strain hardening will change the magnitude of the peak stress where this transition occurs. Finally, the rate of increase/decrease of $\Gamma_{\mathrm{ss}}$ with respect to crack velocity is quite sensitive to

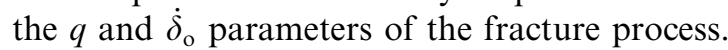

One last feature of the crossover described above is that it does not occur at the same level of $\hat{\sigma} / \sigma_{y}$ for every crack velocity. In other words, there exists a small range of $\hat{\sigma} / \sigma_{y}$ where the toughness initially increases but then decreases with increasing crack velocity. As an example, for $\hat{\sigma} \approx 2.9 \sigma_{y}$ Fig. 6 shows that $\Gamma_{\mathrm{ss}}$ increases in the range of $\dot{a} /\left(\dot{\varepsilon}_{0} R_{0}\right)=0-10$ but then decreases for $\dot{a} /\left(\dot{\varepsilon}_{0} R_{0}\right)=10-1000$. This non-monotonic toughness-velocity behavior occurs in many polymeric materials and is discussed in detail in Webb and Aifantis (1995). This is an interesting prediction of the model, however we note that transitions in the toughness-velocity behavior are more likely to occur due to changes in the fracture process. Along these lines Freund and Lee (1990) demonstrate such a transition by comparing ductile and brittle fracture criteria in a rate dependent strip 
yield model. Also, another ductile to brittle transition was illustrated with the micromechanical model presented in Section 6.

Finally, void cell simulations were performed to elucidate the fracture process in rubber toughened epoxies and to compliment the predictions of the EPZ fracture model. As demonstrated by the model results of Section 5 the predicted macroscopic fracture toughness is quite sensitive to the rate properties of the fracture process, $q$ and $\dot{\delta}_{\mathrm{o}}$. This sensitivity allows us, to some extent, to determine some features, e.g. peak stress and relative rate sensitivity, of the fracture process based on macroscopic toughness measurements. More detailed understanding and fine tuning of the fracture process parameters is then obtained from physically based micromechanical models.

\section{Acknowledgements}

This work was supported in part by NSF Grants CMS-96-34632 and DMR-94-00396 and in part by the Division of Engineering and Applied Sciences, Harvard University. T.P. acknowledges a fellowship from the Fonds National de la Recherche Scientifique (FNRS) Belgium, and postdoctoral fellowships from the Belgian American Educational Foundation Inc. (BAEF) and from the Université catholique de Louvain (UCL).

\section{References}

ABAQUS, 1997. ABAQUS Theory Manual. Hibbit, Karlsson \& Sorenson.

Andersson, H., 1977. Analysis of a model for void growth and coalescence ahead of a moving crack tip. J. Mech. Phys. Solids 25, 217-233.

Boyce, M.C., Parks, D.M., Argon, A.S., 1988. Large inelastic deformation of glassy polymers part I: rate dependent constitutive model. Mech. Mater. 7, 15-33.

Bradley, W., Cantwell, W.J., Kausch, H.H., 1998. Viscoelastic creep crack growth: a review of fracture mechanical analyses. Mech. Time-Dep. Mater. 1, 241-268.

Crocombe, A.D., Richardson, G., Smith, P.A., 1995. A unified approach for predicting the strength of cracked and noncracked adhesive joints. J. Adhesion 49, 211-244.

Dean, R.H., Hutchinson, J.W., 1980. Quasi-static steady crack growth in small-scale yielding. In: Fracture Mechanics: 12th
Conference, ASTM STP 700, American Society for Testing and Materials, pp. 383-405.

Dhirendra, V.K., Narasimhan, R., 1998. Mixed-mode steadystate crack growth in elastic-plastic solids. Engng. Fract. Mech. 59, 543-559.

Doll, W., 1983. Optical interference measurements and fracture mechanics analysis of crack tip craze zones. Adv. Polym. Sci. 52\&53, 105-168.

Drugan, W.J., Rice, J.R., Sham, T.-L., 1982. Asymptotic analyses of growing plane strain tensile cracks in elasticideally plastic solids. J. Mech. Phys. Solids 30, 447-473.

Du, J., Thouless, M.D., Yee, A.F., 1998. Development of a process zone in rubber-modified epoxy polymers. Int. J. Fract. 92, 271-285.

Du, J., Thouless, M.D., Yee, A.F., 2000. Effects of rate on crack growth in a rubber-modified epoxy, Acta Mater. (submitted).

Estevez, R., Tijssens, M.G.A., Van der Giessen, E., 1999. Modeling of the competition between shear yielding and crazing on glassy polymers. Report No. 1216, Delft University of Technology.

Freund, L.B., Lee, Y.J., 1990. Observations on high strain rate crack growth based on a strip yield model. Int. J. Fract. 42, 261-276.

Gurson, A.L., 1977. Continuum theory of ductile rupture by void nucleation and growth: part I - yield criteria and flow rules for porous ductile media. J. Engng. Mater. Technol. 99, 2-15.

Huang, Y., Kinloch, A., 1992. Modelling of the toughening mechanisms in rubber-modified epoxy polymers. J. Mater. Sci. 27, 2763-2769.

Kinloch, A.J., Gilbert, O.G., Shaw, S.J., 1986. A mechanism for ductile crack growth in epoxy polymers. J. Mater. Sci. 21, 1051-1056.

Kramer, J.K., Berger, L.L., 1990. Fundamental processes of craze growth and fracture. Adv. Polym. Sci. 91/92, 1-68.

Lam, P.S., McMeeking, R.M., 1984. Analyses of steady quasistatic crack growth in plane strain tension in elasticplastic materials with non-isotropic hardening. J. Mech. Phys. Solids 32, 395-414.

Lazzeri, A., Bucknall, C.B., 1995. Applications of a dilatational yielding model to rubber-toughened polymers. Polymer 36, 2895-2902.

Marusich, T.D., Ortiz, M., 1995. Modelling and simulation of high-speed machining. Int. J. Numer. Meth. Engng. 38, 3675-3694.

Miao, Y., Drugan, W.J., 1995. Asymptotic analysis of growing crack stress/deformation fields in porous ductile metals and implications for stable crack growth. Int. J. Fract. 72, 6996.

Parks, D.M., Lam, P.M., McMeeking, R.M., 1981. Some effects of inelastic constitutive models on crack tip fields in steady quasistatic growth. Advances in Fracture Research, In: Francois, D. (Ed.), Fifth International Conference on Fracture, Cannes, France, 5, pp. 2607.

Pearson, R.A., Yee, A.F., 1986. Toughening mechanisms in elastomer-modified epoxies. J. Mater. Sci. 21, 2475-2488. 
Pearson, R.A., Yee, A.F., 1991. Influence of particle size and particle size distribution on toughening mechanisms in rubber-modified epoxies. J. Mater. Sci. 26, 3828-3844.

Sener, J.Y., 1999. Développement de Nouvelles Méthodologies de Mesure de l'Adhésion d'Assemblages Collés et de Revêtements sur Substrats Métalliques, Ph.D. Thesis, Université catholique de Louvain.

Tvergaard, V., Hutchinson, J.W., 1992. The relation between crack growth resistance and fracture process parameters in elastic-plastic solids. J. Mech. Phys. Solids 40, 13771397.

Webb, T.W., Aifantis, E.C., 1995. Oscillatory fracture in polymeric materials. Int. J. Solids Struct. 32, 2725-2743.

Wei, Y., Hutchinson, J.W., 1997a. Nonlinear delamination mechanics for thin films. J. Mech. Phys. Solids 45, 11371159.
Wei, Y., Hutchinson, J.W., 1997b. Steady-state crack growth and work of fracture for solids characterized by strain gradient plasticity. J. Mech. Phys. Solids 45, 1253-1273.

Worswick, M.J., Pick, R.J., 1990. Void growth and constitutive softening in a periodically voided solid. J. Mech. Phys. Solids 38, 601-625.

Wu, P.D., Van der Giessen, E., 1993. On improved network models for rubber-elasticity and their application to orientation hardening in glassy polymers. J. Mech. Phys. Solids 41, 427-456.

Xia, L., Shih, C.F., 1995. Ductile crack growth. II. Void nucleation and geometry effects on macroscopic fracture behaviour. J. Mech. Phys. Solids 43, 1953-1981.

Yee, A.F., Li, D., Li, X., 1993. The importance of constraint relief caused by rubber cavitation in the toughening of epoxy. J. Mater. Sci. 28, 6392-6398. 